\title{
Temporal characterization of a self-modulated laser wakefield
}

\author{
S. P. Le Blanc ${ }^{\dagger}$, M. C. Downer ${ }^{\dagger}$, R. Wagner, \\ S.-Y. Chen, A. Maksimchuk, G. Mourou, and D. Umstadter \\ Center for Ultrafast Optical Science, University of Michigan, Ann Arbor, MI 48109 \\ ${ }^{\dagger}$ Department of Physics, The University of Texas at Austin, Austin, Texas 78712
}

\begin{abstract}
The temporal envelope of plasma density oscillations in the wake of an intense $\left(\mathrm{I} \sim 4 \times 10^{18} \mathrm{~W} / \mathrm{cm}^{2}, \lambda=1 \mu \mathrm{m}\right.$ ) laser pulse (400 $\left.\mathrm{fs}\right)$ is measured using forward Thomson scattering from a copropagating, frequency doubled probe pulse. The wakefield oscillations in a fully ionized helium plasma $\left(\mathrm{n}_{e}=3 \times 10^{19} \mathrm{~cm}^{-3}\right)$ are observed to reach maximum amplitude $\left(\delta \mathrm{n}_{e} / \mathrm{n}_{e} \sim\right.$ $0.1) 300 \mathrm{fs}$ after the pump pulse. The wakefield growth $\left(4 \mathrm{ps}^{-1}\right)$ and decay $\left(1.9 \mathrm{ps}^{-1}\right)$ rates are consistent with the forward Raman scattering instability and beam loading, respectively.
\end{abstract}

\section{Introduction}

Because electrostatic fields in a plasma wave $(E \geq 100 \mathrm{GV} / \mathrm{m})$ can exceed by three orders of magnitude those in conventional RF linacs, plasma based accelerators can potentially offer a compact method for accelerating high energy electron pulses for the next generation of collider experiments [1]. However, particle beams accelerated by plasma based accelerators must meet challenging requirements, including high luminosity, low emittance, and high beam loading efficiency. Of the several methods for driving large amplitude plasma waves, the laser wakefield accelerator (LWFA) [1] and its variant, the self-modulated LWFA $[1,2,3,4,5,6]$, have recently received considerable attention because the the terawatt class laser systems [7] needed to drive the wakefield have been significantly reduced in size. In the LWFA, the amplitude $\alpha=\delta n_{e} / n_{e}$ of the plasma wave 
can be resonantly excited by the ponderomotive force of the laser pulse if the laser pulse duration is approximately half of the plasma wave period $\tau_{p}=2 \pi / \omega_{p}$, where $\omega_{p}=\sqrt{4 \pi e^{2} n_{e} / m_{e}}$ is the electron plasma frequency and $n_{e}$ is the plasma density.

For the self-modulated LWFA, the plasma density is chosen to be much larger than for the standard LWFA so that the forward Raman scattering (FRS) instability can grow. The FRS instability is the conversion of an electromagnetic wave $\left(\omega_{o}, k_{o}\right)$ into a plasma wave $\left(\omega_{p}, k_{p}\right)$ and Stokes $\left(\omega_{o}-\omega_{p}, k_{o}-k_{p}\right)$ and anti-Stokes $\left(\omega_{o}+\omega_{p}, k_{o}+k_{p}\right)$ electromagnetic side bands [8]. Electron density perturbations in the plasma cause local variations in the group velocity $v_{g}=c\left(1-\omega_{p}^{2} / w_{o}^{2}\right)^{1 / 2}$ of the laser pulse. As a result, light that propagates near a density maximum (minimum) will slow down (speed up). Eventually, the light is bunched to positions where $\delta n_{e}=0$. Because the plasma density perturbation and the bunching of the light are $\pi / 2$ out of phase, the ponderomotive force of the bunched light will reinforce the original density perturbation. Since the maximum longitudinal electric field $E_{z}$ scales as $E_{z} \propto \alpha \sqrt{n_{e}}$, the self-modulated wakefield can produce a much larger accelerating field than the standard LWFA. If the plasma wave is driven to wavebreaking amplitudes, then background electrons can be trapped and accelerated in the propagation direction of the laser pulse $[3,4,5,6]$.

In these proceedings, we report time resolved measurements of the amplitude of the self-modulated laser wakefield obtained using forward Thomson scattering from a co-propagating, frequency doubled probe pulse [9]. In addition to measuring the growth and decay rate of the wakefield, we report that the onset of FRS is consistent with a plasma density perturbation driven by the ionization front of the laser pulse. These observations are important for testing recently developed $2 \mathrm{D}$ particle-in-cell (PIC) simulations of laser plasma interactions $[10,11,12]$ and for the design of plasma based accelerators. Although future plasma based accelerators are likely to be based on the standard LFWA scheme, the present investigation of a self modulated LFWA nevertheless provides a useful testbed to investigate plasma instabilities and beam loading issues for atmospheric density plasmas.

\section{Experimental setup}

In the present experiments, a hybrid Ti:Sapphire - Nd:Glass laser system capable of delivering $3 \mathrm{~J}, 400 \mathrm{fs}$ laser pulses was used to drive the self modulated LWFA. The $43 \mathrm{~mm}$ diameter beam was focused by an f/4 off-axis parabolic mirror to a spot size ( $\mathrm{e}^{-2}$ intensity) of $r_{o}=8.9 \mu \mathrm{m}$, giving a maximum vacuum intensity 
of $\mathrm{I}=6 \times 10^{18} \mathrm{~W} / \mathrm{cm}^{2}$. The laser was focused onto a supersonic helium gas jet whose neutral density varied linearly with backing pressure [6]. The neutral gas density produced by the nozzle was measured to have a flat top shape with a width of $500 \mu \mathrm{m}$ and $200 \mu \mathrm{m}$ gradients on each side. To probe the lifetime of the plasma wave, a small portion (20\%) of the infrared laser pulse was split off, frequency doubled in a $4 \mathrm{~mm}$ Type I KDP crystal, and then made to copropagate with the infrared pump pulse. The temporal overlap between the IR pump pulse and orthogonally polarized green probe pulse was measured with a resolution of $\pm 100 \mathrm{fs}$ by frequency domain interferometry. The probe pulse had a maximum energy of $15 \mathrm{~mJ}$ and could be focused to a spot size of $6.4 \mu \mathrm{m}$ by the harmonically coated parabolic mirror. Forward scattered light from the probe pulse was collected on axis, passed through a polarizer to suppress scattered pump light, and measured with a prism spectrometer which has a resolution of $\lambda / \Delta \lambda=$ 600 at $\lambda=1.053 \mu \mathrm{m}$.

\section{Forward Raman scattering}

When the peak power of the IR pump pulse ( $P \geq 1 \mathrm{TW}$ ) is near the critical power for relativistic self-focusing $\mathrm{P}_{c}=17\left(\omega_{o}^{2} / \omega_{p}^{2}\right) \mathrm{GW}$, the forward scattered light from the pump pulse shows the appearance of three anti-Stokes Raman shifted side bands separated by the plasma frequency $\left(\omega_{p} \sim 3 \times 10^{14} \mathrm{~s}^{-1}\right)$. [6]. Numerical simulations indicate that the appearance of multiple side bands is clear evidence of FRS. From the relative amplitude of the Raman satellites, the plasma wave amplitude is estimated to be $\delta n / n=0.08-0.4$, depending on the pump power and plasma density [6]. Under these conditions, a collimated beam of $2 \mathrm{MeV}$ electrons with a transverse emittance of $1 \mathrm{~mm}$ mrad is emitted in the laser propagation direction [6].

In one dimension, the spatio-temporal growth rate for the FRS instability starting from a uniform noise source $\delta n_{s}$ is given by [10]:

$$
\delta n= \begin{cases}\delta n_{s} \cosh \left(\gamma_{o} \tau\right) & \psi \geq \tau c \\ \delta n_{s} \sum\left(\frac{\psi / c}{\tau-\psi / c}\right)^{n} I_{2 n}\left(2 \gamma_{o} \sqrt{(\tau-\psi / c) \psi / c}\right) & \tau c \geq \psi\end{cases}
$$

where $I_{2 n}$ is the modified Bessel function of the first kind, $\psi$ is the distance from the leading edge of the pulse, $\tau$ is the propagation time, $a_{o}$ is the normalized vector 
potential of the laser $\left(a_{o}=8.5 \times 10^{-10} \lambda(\mu \mathrm{m})\left(I\left(\mathrm{~W} / \mathrm{cm}^{2}\right)^{1 / 2}\right)\right.$, and

$$
\gamma_{o}=\frac{\omega_{p}^{2}}{\sqrt{8} \omega_{o}} \frac{a_{o}}{\left(1+a_{o}^{2} / 2\right)^{1 / 2}}
$$

is the the temporal growth rate. Because the laser pulse changes shape as the instability grows with distance, FRS for relativistic intensity laser pulses is a highly nonlinear process.

When the green probe pulse propagates through the plasma, collective Thomson scattering from the wakefield plasma wave causes multiple side bands to appear in the spectrum of the forward scattered probe light. For $P=3$ TW and a backing pressure of 100 psi, Fig. 1 shows the appearance of first and second order Thomson scattered satellites which are separated by the plasma frequency $\omega_{p}=2.7 \times 10^{14} \mathrm{~s}^{-1}$. The amplitude of the plasma wave can be determined from both the absolute and relative scattering efficiency of the Thomson side bands. For collective Thomson scattering, the absolute scattering efficiency is given by [13]:

$$
\frac{P_{s}}{P_{o}}=\frac{1}{4}\left(\delta n_{e}\right)^{2} r_{o}^{2} \lambda_{o}^{2} L^{2} \frac{\sin ^{2}(\Delta \mathrm{kL})}{(\Delta k L)^{2}}
$$

where $r_{o}$ is the classical electron radius, $\lambda_{o}$ is the wavelength of the incident light, $\mathrm{L}$ is the interaction length, $\Delta k=k_{o}-k_{s} \pm k_{p}$ is the wavevector mismatch, $k_{p}=$ $\omega_{p} / v_{p}$, and the phase velocity $v_{p}$ of the plasma wave is equal to $v_{g}$ of the pump pulse. For direct forward scatter of the first anti-Stokes sideband $\left(\Delta k=8 \times 10^{3}\right.$ $\left.\mathrm{m}^{-1}\right)$ and $\mathrm{L}$ equal to the confocal beam parameter $(\mathrm{L}=430 \mu \mathrm{m})$, the phase mismatch factor $F=\sin ^{2}(\Delta \mathrm{kL}) /(\Delta \mathrm{kL})^{2}=0.02$ and the amplitude of the plasma wave is determined to be $\alpha=0.08$. Similarly, from the first Stokes line, $\alpha=0.06$. Applying the same analysis to the second order satellites, the amplitude of the second harmonic of the plasma wave is $\delta n_{2} / n=0.01$. From harmonic wave analysis [14], the fundamental amplitude of the plasma wave is related to its second harmonic by $\alpha \sim\left(\delta n_{2} / n\right)^{1 / 2}$, or $\alpha=0.1$ for the present conditions at 100 psi backing pressure. Using harmonic analysis and Eq. 2, the relative amplitude of the first and second order Thomson satellites can be used to determine $\alpha$ without specifying L: $\alpha \sim\left(P_{2} / P_{1}\right)^{1 / 2}$, or $\alpha=0.1$. Thus, the absolute and relative scattering efficiencies yield plasma wave amplitudes that are in good agreement. For a Gaussian laser focus, the peak amplitude is estimated to be $\alpha_{p}=0.2$. 


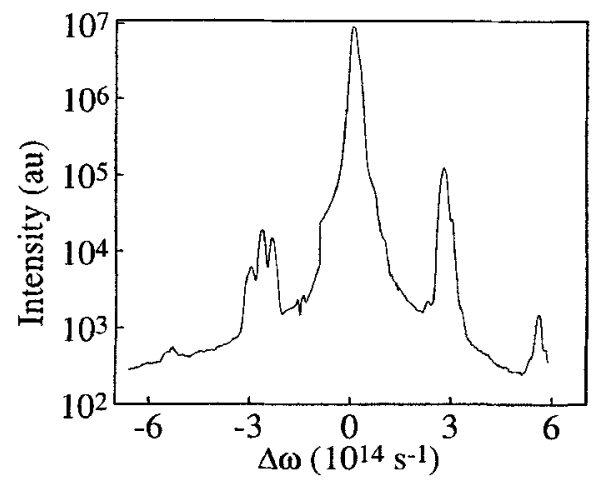

Figure 1: Spectrum of the Thomson scattered probe light for a helium backing pressure of $100 \mathrm{psi}, \mathrm{P}_{\text {pump }}=3 \mathrm{TW}$, and $\Delta t=0$.

\section{Wakefield temporal characterization}

By measuring Thomson scattering from the probe pulse as a function of the delay between the pump and probe, the temporal envelope of the wakefield oscillations can be recorded. Fig. 2a shows the change in the spectrum of the probe pulse as the delay $(\Delta \mathrm{t})$ between a $600 \mathrm{~mJ}(1.5 \mathrm{TW})$ pump and a $15 \mathrm{~mJ}$ probe pulse is varied from -1 to 3 ps. Only the first order Thomson scattered satellites are shown. When the Thomson scattered satellites first become observable $(\Delta t \sim-700 \mathrm{fs})$, their frequency shift is $\Delta \omega=3 \times 10^{14} \mathrm{~s}^{-1}$. The frequency shift then increases gradually and becomes fixed at $\Delta \omega=3.3 \times 10^{14} \mathrm{~s}^{-1}$ for $\Delta t \geq 0$, because it takes a finite time for the helium to become fully ionized. Simultaneously, a blue shifted wing appears on the green probe pulse because of the rapid increase in the electron density [15]. This temporal position of the ionization front is consistent with a calculation of field ionization of helium for our pump pulse parameters.

At most delay times, the amplitude of the anti-Stokes satellite is larger than that of the Stokes due to more favorable phase matching of the anti-Stokes for direct forward scatter. Using the scattering efficiencies of the Stokes and antiStokes sidebands and Eq. 3, Fig. $2 \mathrm{~b}$ shows the plasma wave amplitude as function of the probe delay time. The plasma wave is measured to have a peak amplitude $\alpha=0.1$, which corresponds to a maximum longitudinal field of $E_{z}=56 \mathrm{GV} / \mathrm{m}$ for a cold, nonrelativistic fluid. Under the current tight focusing conditions, the maximum radial electric field is $E_{r}=2 E_{z} / k_{p} r_{o}=0.2 E_{z}$. Large radial fields can 

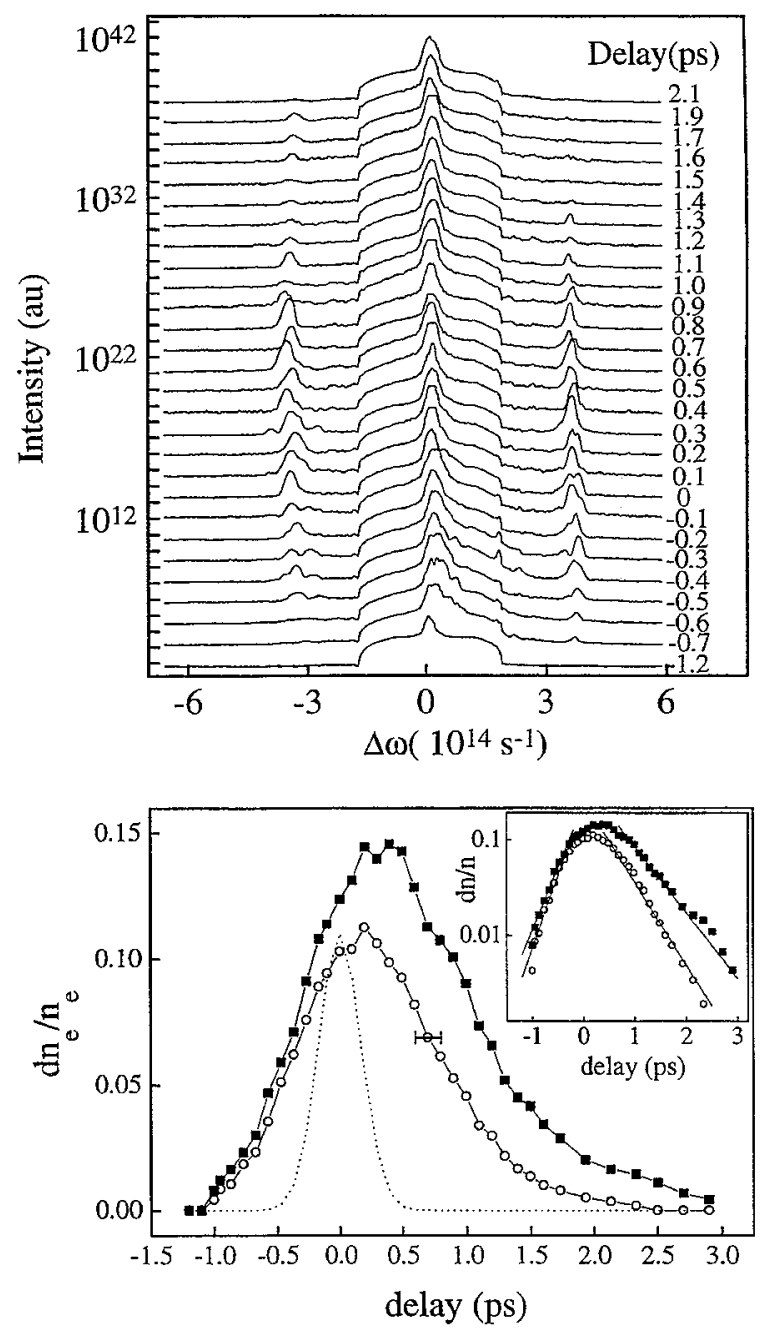

Figure 2: a) Forward scattered probe spectra at a helium backing pressure of 180 psi as a function of the delay between the $1.5 \mathrm{TW}$ pump. b) Plasma wave amplitude determined from the scattering efficiency of the Stokes (filled squares) and anti-Stokes (open circles) satellites as a function of delay. The dotted line indicates the 400 fs pump pulse. The inset shows the exponential fits for the wakefield growth and decay. 
give rise to an increase in the transverse emittance of the accelerated electron beam. Fig. $2 \mathrm{~b}$ shows that the wakefield amplitude maximizes at the end of the pump pulse ( $\Delta t=300 \pm 100 \mathrm{fs}$ ) and lasts for approximately 2 ps longer. The leading edge of the wakefield rises sharply due to the exponential growth rate of FRS. Note that the probe pulse duration $(\sim 300 \mathrm{fs})$ is much longer than the plasma period $\left(\tau_{p}=21 \mathrm{fs}\right.$ for $n_{e}=3 \times 10^{19} \mathrm{~cm}^{-3}$ ) in the present experiment. Therefore, we do not resolve individual wakefield oscillations [16].

\subsection{Wakefield growth}

As shown in Fig. $2 b$, the onset of the plasma wave occurs $\sim 1$ ps before the peak of the pump pulse. This observation is contrary to recent $2 \mathrm{D}$ simulations which show the plasma wave growing closer to the peak of the pulse $[10,12]$. In the simulations, the onset of FRS occurs when the leading edge of the pulse is steepened by backward Raman scattering and pump depletion. In the present experiments, FRS starts near the position of the ionization front. The ponderomotive force from an ionization front can create a large amplitude noise source to seed FRS [17, 18]. The ionization front travels at the phase velocity of the plasma wave and creates a noise source that scales as $\delta n_{s i} / n \sim a_{o}^{2}\left(\psi_{i}\right) / 4$, where $\psi_{i}$ is the position in the pulse where the ionization threshold occurs [19]. For single and double ionization of helium, $\delta n_{s i} / n=10^{-4}$ and $10^{-3}$, respectively, which is larger than the noise source due to intensity gradients of the laser pulse $\left(\delta n_{s} / n \sim 10^{-6}\right)$ [10]. Consequently, future numerical simulations should include ionization effects.

From the measurements in Fig. $2 b$, the wakefield growth and decay rates can be determined. Fitting an exponential to the growth of the plasma wave, the deconvolved growth rate is determined to be $3.5 \pm 0.3 \mathrm{ps}^{-1}$ from the anti-Stokes Thomson signal, and $3.3 \pm 0.3 \mathrm{ps}^{-1}$ from the Stokes signal. For the current experimental parameters $\left(\omega_{p}=3.3 \times 10^{14} \mathrm{~s}^{-1}, \mathrm{a}_{o}=1\right)$, the temporal growth rate of the forward Raman scattering instability $\left(\gamma_{o}=18 \mathrm{ps}^{-1}\right)$ is over estimated if the peak intensity of the laser pulse is substituted in the expression for $\gamma_{o}$, since the theoretical derivation for $\gamma_{o}$ assumes a constant amplitude pulse. In reality, exponential growth actually takes place during the leading edge of the pulse where $a_{o} \ll 1$. For the value of $a_{o}$ that is $400 \mathrm{fs}$ before the pump pulse maximum, $\gamma_{o}=8$ $\mathrm{ps}^{-1}$. Since the Rayleigh time $\tau_{r}=z_{r} / c$ is longer than the pulse width $\psi / c$ in Eq. 1 , the growth of FRS is approximately given by $\delta n=\delta n_{s} I_{o}\left(2 \gamma_{o} \sqrt{\psi \tau / c}\right)$. Evaluation of the Bessel function gives a rise time of $9 \mathrm{ps}^{-1}$ when $\mathrm{a}_{o}$ is allowed to follow the temporal pulse shape and the interaction length is equal to $2 z_{r}$. A number of factors can cause the measured growth rate to be slightly lower than the calcu- 
lated growth of four wave resonant FRS. Because FRS is a convective instability, the actual growth rate changes at each delay time (or position $\psi$ ). Furthermore, FRS evolves into different regimes - four wave nonresonant, three wave, and selfmodulation - as time progresses, each with its own growth rate [19]. For example, the ratio for $1 \mathrm{D}$ growth of FRS $\left(\Gamma_{1 D}\right)$ to that for 3D self modulation $\left(\Gamma_{3 D}\right)$ is given by : $\Gamma_{1 D} / \Gamma_{3 D}=\left(k_{p}^{2} r_{o}^{2} / 2 k_{o}^{2}\right)^{1 / 3}$ [4]. In the present experiments, this ratio is unity, so 3D instabilities will compete with ID FRS. Since the plasma wavelength $\left(\lambda_{p} \sim 6 \mu \mathrm{m}\right)$ is comparable to $r_{o}$, the plasma wave is near the 3D limit where it has been shown that ID FRS cannot be resonantly driven because of the complicated three dimensional shape of the plasma wave. $[10,12]$.

To further investigate the growth of the wakefield, Thomson scattering was measured as a function of the gas jet backing pressure and the peak power of the pump pulse. Fig. 3a shows variation in the Thomson spectra for a fixed laser power $(\mathrm{P}=3 \mathrm{TW})$ as the helium backing pressure varies from 30 to $180 \mathrm{psi}$. As expected, the frequency separation between the satellites, given by $\omega_{p}$, increases as the square root of the backing pressure. For 3 TW pump power, the first antiStokes Thomson satellite appears for a backing pressure of 40 psi. From the measured frequency shift of the satellite, the critical power for self focusing at this backing pressure is $P_{c}=1.4 \mathrm{TW}$, and thus $\mathrm{P} / \mathrm{P}_{c}=2.1$ and $\gamma_{o}=9 \mathrm{ps}^{-1}$. In a similar pressure scan conducted at $\mathrm{P}=1.7 \mathrm{TW}$, the first Thomson satellite appeared at a backing pressure of $80 \mathrm{psi}$ where $P / P_{c}=1.9$ and $\gamma_{o}=10 \mathrm{ps}^{-1}$. As the peak power of the pump pulse increases, Fig. $3 \mathrm{~b}$ shows the change in the Thomson spectra for a fixed backing pressure (180 psi) and at a fixed delay time $(\Delta t=0)$. The first Thomson scattered satellite appears at $\mathrm{P}=0.78 \mathrm{TW}$, or where $\mathrm{P} / \mathrm{P}_{c}=1.7$ and $\gamma_{o}=14$ $\mathrm{ps}^{-1}$. At the threshold for Thomson scattering, the ratio $\mathbf{P} / \mathrm{P}_{c}$ decreases as the backing pressure (and hence electron density) increases. This trend is evident from Eq. 1 which indicates that the threshold for FRS depends on $\omega_{p} / \omega_{o}$ and $\mathrm{P} / \mathrm{P}_{c}[8]$. For both the pressure and power scan in Fig. 3, $\gamma_{o} \sim 10 \mathrm{ps}^{-1}$ even though $P / P_{c}$ and the backing pressures are different at threshold. The growth rate of the plasma wave as determined by the FRS threshold measurements is in reasonable agreement with the rate obtained from the delay scan in Fig $2 b$. These observations are also in agreement with those of Ref. [6] which showed that Raman satellites from the pump pulse first appear at $P / P_{c}=0.5$ and $\gamma_{o}=6 \mathrm{ps}^{-1}$ for a helium backing pressure of 150 psi. This indicates that our experiment is in the multidimensional regime of self-modulation [21,22], as distinguished from previous experiments $[3,4,5]$ which were interpreted within the context of ID FRS.

As the laser power increases, Fig. $3 \mathrm{~b}$ shows that the width of the sidebands 

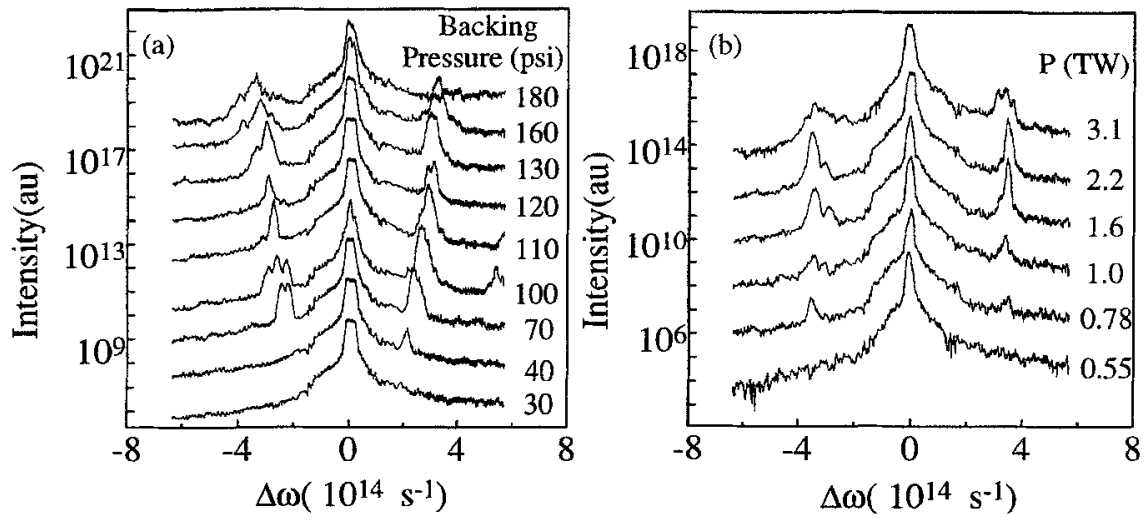

Figure 3: a) Pressure dependence of the Thomson scattered probe light for $\mathrm{P}=3$ TW and $\Delta t=0$. b) Variation in Thomson scattering with pump pulse power for a fixed backing pressure of 180 psi and $\Delta t=0$.

increases by a factor of 2 , indicating a loss of coherence of the plasma wave. Although sideband broadening has been reported and attributed to wavebreaking [5], the present observations and Ref. [6] are the first showing broadening and spectral modulation of both Thomson and Raman side bands. A number of processes could give rise to the modulations observed on the scattered sidebands. The high frequency modulations may come from phase matching effects in the scattering efficiency. However, the frequency modulation due the $\operatorname{sinc}^{2}$ factor in Eq. $3\left(\delta \omega_{\text {sinc }} \sim 10^{12} \mathrm{~s}^{-1}\right)$ is smaller than the observed modulation frequency $\delta \omega=3 \times 10^{13} \mathrm{~s}^{-1}$. Similar modulation has been observed in backscattered Raman spectra and attributed to ion plasma wave fluctuations [20]. Lastly, simulations show that relativistic self phase modulation can produce spectral modulations with the experimentally observed frequency modulation. However, experimental evidence for this mechanism is inconclusive thus far.

\subsection{Wakefield decay}

The decay of the wakefield is caused by the conversion of collective plasma wave energy into particle energy. In Fig. $2 b$, the exponential decay rate of the wakefield is $1.6 \pm 0.1 \mathrm{ps}^{-1}$ and $1.9 \pm 0.2 \mathrm{ps}^{-1}$ as determined from the Stokes and antiStokes Thomson signals, respectively. If the observed damping of the wakefield is 
due to beam loading [23], then the energy in the plasma wave should be transferred to the accelerated electrons. The energy of the plasma wave is approximately given by : $W_{\text {wave }}=\varepsilon_{o} E_{z}^{2} A L / 2$, where $A=\pi r_{o}^{2}$ and $L=2 z_{r}$. For $\alpha=0.1, \mathrm{~W}_{\text {wave }}=$ $1.6 \mathrm{~mJ}$. Since this compares well to the energy of $10^{9}$ electrons being accelerated to $2 \mathrm{MeV}$, or $\mathrm{W}_{\text {beam }}=1 \mathrm{~mJ}$, it is likely that beam loading and particle acceleration contribute to wakefield decay.

A complementary way of discussing beam loading is by the concept of Landau damping of trapped electrons. Such trapped electrons come from hot electrons generated by Raman back-and side-scatter, and above threshold ionization (ATI). For $a_{o}=1$, ATI plasma heating is estimated to yield a plasma with $T_{e} \sim$ $80 \mathrm{keV}$ [24], while Raman back- and side scattering generate hot electrons with $T_{e} \sim 4 \mathrm{keV}$ [25] and $T_{e} \geq 10 \mathrm{keV}$ [26], respectively. If the damping of the plasma wave is dominated by the acceleration of trapped electrons, the rate of decrease of $W_{\text {wave }}$ must be equal and opposite to the rate of increase of $W_{\text {beam }}$. From linear theory [27], the Landau damping rate for trapped particles is approximately equal to $\gamma_{L}=L_{d} / c$, where $L_{d}$ is the dephasing length. Under the present conditions, $\gamma_{L}=2 \mathrm{ps}^{-1}$, which is consistent with the measured wakefield decay rate of $1.9 \mathrm{ps}^{-1}$. Thus, the wakefield decays on the time scale that trapped particles exchange energy with the plasma wave. Damping of relativistic plasma waves due to nonlinear Landau damping has also been observed in simulations of the resonant laser plasma accelerator [28].

Beam loading can also be analyzed by considering the superposition of the laser induced wakefield and the wakefield generated by the accelerated particle bunch itself [23]. The maximum number of particles that can be accelerated is approximately given by the number of electrons required for the electron bunch generated wakefield to cancel the laser induced wakefield. For a short electron bunch, the maximum number of particles $N_{\max }$ is given by [23]:

$$
N_{\max }=5 \times 10^{5} \alpha A \sqrt{\left(n_{o}\right)}
$$

where $n_{o}$ is the electron density in $\mathrm{cm}^{-3}$ and $\mathrm{A}$ is the effective cross sectional area of the beam in $\mathrm{cm}^{2}$. Under the present conditions of $n_{o}=3.3 \times 10^{19} \mathrm{~cm}^{-3}, \mathrm{~A}=\pi r_{o}^{2}$, and $\alpha=0.15, \mathrm{~N}_{\max }=10^{9}$, which is in good agreement with the measured value of $2 \times 10^{9}$ particles. If $\mathbf{N}_{\max }$ particles are loaded into a given acceleration phase of the wakefield, all subsequent wakefield oscillations will be cancelled since all of the wave energy is absorbed by the particles. Experimentally, the wakefield is observed to damp over many wakefield oscillation periods $\left(\tau_{p} \sim 20 \mathrm{fs}\right)$. Consequently, the laser induced wakefield is diminished by contributions from many 
accelerated particle bunches, each of which contains a number of particles $N_{b}$ less than $N_{\max }$. Since the number of acceleration buckets $b$ occupied during the decay of the wakefield is $b \sim 2(p s) / \tau_{p} \sim 100$, the number of accelerated particles in each bunch is approximately $N_{b}=N_{\max } / b=10^{7}$ particles if it is assumed that each particle bunch contributes equally to the wakefield decay.

\section{Summary}

In summary, we have determined that the rise time of the self-modulated wakefield is in agreement with that expected from the growth of the FRS instability and that the decay of the wakefield is consistent with beam loading or Landau damping of the plasma wave. The onset of FRS is consistent with an ionization front induced plasma wakefield, and the detection of a large amplitude plasma wave by Thomson scattering occurs near the critical power for relativistic selffocusing. Channeling of the laser pulse due to relativistic self-focusing was not conclusively observed in the present experiments since the plasma length (defined by the width of the gas jet) was nearly equal to the confocal parameter of the laser beam. Using a gas jet that produced a longer interaction length, channeling of the laser pulse was recently observed over $750 \mu \mathrm{m}$ [29].

\section{Acknowledgements}

This work was supported by DOE Grant No. DEFG03-96-ER-40954, DOE/LLNL subcontract No. B307953, and the NSF STC PHY 8920108. M. C. D. acknowledges a Faculty Research Assignment from the University of Texas.

\section{References}

[1] T. Tajima and J. Dawson, Phys. Rev. Lett 43, 267 (1979)

[2] P. Sprangle et al., Phys. Rev. Lett 69, 2200 (1992); T. M. Antonsen and P. Mora, Phys. Rev. Lett 69, 2204 (1992); N. E. Andreev et al., JETP Lett., 55, 571 (1992).

[3] C. A. Coverdale et al., Phys. Rev. Lett 74, 4659 (1995).

[4] K. Nakajima et al., Phys. Rev. Lett 74, 4428 (1995) 
[5] A. Modena et al., Nature 377, 606 (1995).

[6] D. Umstadter et al., Science 273, 472 (1996).

[7] P. Maine et al., IEEE J. Quantum Electron. 24, 398 (1988).

[8] W. B. Mori et al., Phys. Rev. Lett 72, 1482 (1994).

[9] C. I. Moore et al., Bullet. Amer. Phys. Soc. 40, 1797 (1995); A. Ting et al., these proceedings.

[10] C. D. Decker et al., IEEE Trans. Plasma Sci. 24, 379 (1996); C. D. Decker et al., Phy. Rev. E. 50, R3338 (1994).

[11] S. V. Bulanov et al. Phys. Rev. Lett 74, 710 (1995).

[12] K.-C. Tzeng et al., Phys. Rev. Lett 76, 3332 (1996).

[13] R. E. Slusher and C. M. Surko,Phys. Fluids 23, 472 (1980).

[14] D. Umstadter et al., Phys. Rev. Lett 59 292, (1987).

[15] W. M. Wood et al., Phys. Rev. Lett 67, 3523 (1991).

[16] C. W. Siders et al., Phys. Rev. Lett 76, 3570 (1996); J. R. Marques et al., ibid., 3566 (1996).

[17] W. B. Mori and T. Katsouleas, Phys. Rev. Lett 693495 (1992).

[18] T. Tajima and D. Fisher, Phy. Rev. E. 53, 1844 1996).

[19] C. D. Decker et al., Phys. Plasmas 3, 1360 (1996).

[20] A. Ting et al., Opt. Lett. 21, 1096 (1996).

[21] E. Esarey et al., Phys. Rev. Lett 72, 2887 (1994).

[22] N. E. Andreev et al., Phys. Plasmas 2, 2573 (1995).

[23] T. Katsouleas et al., Particle Accelerators 22, 81 (1987).

[24] B. M. Penetrante and J. N. Bardsley, Phys. Rev. A 433100 (1991).

[25] S. C. Wilks et al.,Phys. Plasmas 2, 264 (1995). 
[26] Y. Kishimoto et al., to be published.

[27] D. R. Nicholson, Introduction to Plasma Theory (Krieger, Florida, 1992).

[28] D. Umstadter et al., "Resonantly driven laser plasma electron accelerators," in Proceedings of the 1994 Workshop on Advanced Accelerator Concepts, P. Schoessow, ed. (AIP No. 335) (American Institute of Physics, New York, 1995).

[29] S.-Y. Chen et al., these proceedings. 